\title{
Astroparticle, Particle and Space Physics, Detectors and Medical Physics Applications
}

Proceedings of the 9th Conference 
This page is intentionally left blank 


\section{Astroparticle, Particle and Space Physics, Detectors \\ and Medical Physics Applications}

Proceedings of the 9th Conference

Villa Olmo, Como, Italy $\quad 17-21$ October 2005

Editors

Michele Barone

Demokritos Athens, Greece

Emilio Borchi

INFN \& University of Florence, Italy

Andrea Gaddi

CERN, Switzerland

Claude Leroy

Université de Montréal, Canada

Larry Price ANL, USA

Pier-Giorgio Rancoita INFN-Milano, Italy

\section{Randal Ruchti}

University of Notre Dame \&

US National Science Foundation, USA 
Published by

World Scientific Publishing Co. Pte. Ltd.

5 Toh Tuck Link, Singapore 596224

USA office: 27 Warren Street, Suite 401-402, Hackensack, NJ 07601

UK office: 57 Shelton Street, Covent Garden, London WC2H 9HE

British Library Cataloguing-in-Publication Data

A catalogue record for this book is available from the British Library.

\section{ASTROPARTICLE, PARTICLE AND SPACE PHYSICS, DETECTORS AND MEDICAL PHYSICS APPLICATIONS \\ Proceedings of the 9th Conference}

Copyright $\odot 2006$ by World Scientific Publishing Co. Pte. Ltd.

All rights reserved. This book, or parts thereof, may not be reproduced in any form or by any means, electronic or mechanical, including photocopying, recording or any information storage and retrieval system now known or to be invented, without written permission from the Publisher.

For photocopying of material in this volume, please pay a copying fee through the Copyright Clearance Center, Inc., 222 Rosewood Drive, Danvers, MA 01923, USA. In this case permission to photocopy is not required from the publisher.

ISBN $981-256-798-4$

Printed in Singapore by Mainland Press 


\section{Organizing Committee:}
E. Borchi
INFN and University of Florence
C. Leroy
University of Montreal
L. Price
ANL
P.G. Rancoita
INFN-Milano (chairman)
R. Ruchti
University of Notre Dame/US National Science Foundation

\section{Industrial Organizing Committee:}
M. Barone
Demokritos Athens
A. Gaddi
CERN Geneva

International Advisory Committee:

D. Abbaneo CERN Geneva

S. Baccaro ENEA-Rome

P. Binko Observatoire de Geneve \& SynSpace SA

A. Breskin Weizmann Institute

A. Capone INFN and University of Rome1

C. Fabjan CERN Geneva

K. Freudenreich ETH-Zurich

S. Giani CERN Geneva

V. Hagopian Florida State University

E. Longo

E. Nappi

INFN and University of Rome1

S. Pospisil

P.L. Riboni

INFN-Bari

CTU Prague

T.J. Ruth

ETHZ-Zurich

D. Saltzberg

TRIUMF

J. Seguinot

UCLA

V. Sossi

College de France, Paris

University of British Columbia, Vancouver

S. Volonté

ESA Paris

C. Waltham

University of British Columbia, Vancouver 


\section{Plenary Session Organizers:}
A. Capone (INFN \& Univ. of Rome1)
Astroparticle Experiments
S. Giani (CERN Geneva)
Symposium on GEANT4 Applica- tions
E. Nappi (INFN-Bari)
L. Price (ANL)
R. Rutchi (Univ. of Notre Dame)
D. Saltzberg (UCLA)
J. Seguinot (College de France)
V. Sossi (Univ. of British Columbia)
S. Volonté (ESA)
Advanced Detectors and Particle Identification
High Energy Physics experiments
Accelerator and Data/Networking Developments
Astroparticle Experiments
Advanced Detectors and Particle Identification
Radiotherapy and Medical Imaging Space experiment

\section{Parallel Session Organizers:}

D. Abbaneo (CERN Geneva)

S. Baccaro (ENEA, Rome)

S. Giani (CERN Geneva)

P. Binko (Observatoire de Geneve \& SynSpace SA)

D. Saltzberg (UCLA)

A. Capone (INFN \& Univ. of Rome1)

C. Fabjan (CERN Geneva)

V. Hagopian (Florida State Univ.)

C. Leroy (Univ. of Montreal)

E. Nappi (INFN-Bari)

J. Seguinot (College de France)

S. Pospisil (CTU, Prague)

L. Price (ANL)

V. Sossi (Univ. of British Columbia)

S. Volonté (ESA)

Tracking Devices

Radiation Damage

Software Applications

Software Applications

Astroparticle Experiments

Astroparticle Experiments

Miniaturization of Particle Detectors

Poster Session

Calorimetry (both sessions)

Advanced Detectors and Particle Identification

Advanced Detectors and Particle Identification

Medical Application

Instrumentation

High Energy Physics Experiments

Radiotherapy and Medical Imaging Space experiment

\section{Secretariat:}
A. Cazzaniga
Centro Volta, Como
N. Tansini
Centro Volta, Como 


\section{PREFACE}

The exploration of the subnuclear world is done through increasingly complex experiments covering a wide range of energy and performed in a large variety of environments going from particle accelerators, underground detectors up to satellites and space laboratory. The achievement of these research programs calls for novel techniques, new materials and new instrumentation to be used in detectors, often of large scale. Therefore, particle physics is at the forefront of technological advance and also leads to many applications. Among these, medical applications have a particular importance due to health and social benefits they bring to the public.

The International Conference on Advanced Technology and Particle Physics is held every two years. The conference returned to the "Centro di Cultura Scientifica A. Volta" for the 2005 Edition. The Conference welcomed about 300 participants. These participants were representing more than 140 institutions from about 30 countries.

The conference allows a regular review of the advances made in all technological aspects of the experiments at various stages: preparation, data taking or upgrade. The open and flexible format of the Conference is conducive to fruitful exchanges amongst participants, shows the progresses made and gives research directions.

The medical sessions gave an interesting example of merging advanced technologies: particle physics and numerical techniques. Industries specialized in advanced technologies were present at the Conference through a presentation of their products (organized by M. Barone and A. Gaddi)

Plenary and parallel sessions covered Advanced Detectors and Particle Identification (organized by E. Nappi and J. Seguinot), Miniaturizing of Particle Detectors (organized by C. Fabjan), Astroparticle Experiments (organized by D. Saltzberg and A. Capone), Calorimetry (organized by C. Leroy), Software Applications (organized by S. Giani and P. Binko), High Energy Physics Experiments (organized by L. Price), Accelerator and Data/Networking Developments (organized by R. Ruchti), Medical Application Instrumentation (organized by S.Pospisil), Radiotherapy and Medical Imaging (organized by V. Sossi), Radiation Damage (organized by S. Baccaro), Space Experiments (organized by S. Volontè), and Tracking Devices (organized by D. Abbaneo). This edition of the conference was combined with a symposium on the applications of the GEANT4 simulation software. The symposium was organized by S. Giani.

The poster session has always received special attention by the organizers of the conference. This session was organized this year by V. Hagopian. The posters were a great success. The number of posters has more than doubled to about 45 since the 8th ICATPP Conference in 2003. Having their own rooms was conducive of numerous discussions by various interested parties. This year, there were several innovations in the poster presentations. First, one afternoon was designated as the Poster Parallel Session and all the presenters requested to be near their poster. Second, it was requested attaching a small picture of the presenter to every poster so that interested 
parties could recognize the person for possible discussions. Many of the presenters also left copies of their paper on the poster board, so interested parties could pick up a copy. Most of the posters were very elaborate with excellent graphics, printed on photographic type glossy paper. The posters were divided into three general categories as follows: 1. Astroparticle, Underground Experiments, Space Physics and Cosmic Rays; 2. High-Energy Physics Experiments, Trackers, Calorimetry, Software and Data Systems; 3. Advanced Detectors and Medical Physics Applications. Each poster submission was reviewed for relevance to the conference and accepted or rejected. The final poster papers accepted for publications have been included in the appropriate section of the Proceedings, as function of their topic. From the quality of the material of the posters, as well as the poster presentation, it is clear that a lot of effort went into the preparation. We would like to thank the poster presenters for their considerable effort that made the posters a truly important part of this meeting.

We would like to thank the staff of the Centro A. Volta for the excellent support provided to the Conference organization at Villa Olmo. In particular, we would like to extend our appreciation and thanks to the Secretariat of Centro di Cultura Scientifica A. Volta for their help and efficiency with the organization of the Conference and its running.

We are particularly indebted to Dr G. Castiglioni (President of Univercomo), who, by providing a sponsorship, allowed the Conference Organizers to largely reduce or waive the registration fees for the $\mathrm{Ph}$.D. students.

The organizers would like to thank the strong support of INFN and ESA which made the conference possible.

Finally, we would like to thank the speakers for the high quality of their contributions and the participants for their enthusiasm in attending the Conference and contribution to the discussions.

\author{
Michele Barone \\ Emilio Borchi \\ Andrea Gaddi \\ Claude Leroy \\ Larry Price \\ Pier-Giorgio Rancoita \\ Randal Ruchti
}

December 2005 
CONTENTS

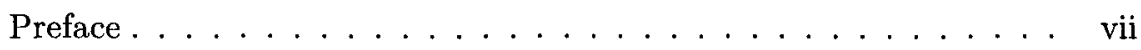

\section{Advanced, Miniaturized Detectors and Particle}

Identification . . . . . . . . . . . . . . . . 1

A Novel Micromegas Detector for In-core Nuclear Reactor Neutron Flux Measurements . . . . . . . . . . . . . . . 3

The ALICE TPC . . . . . . . . . . . . . . 10

The ATLAS RPC Test Stands . . . . . . . . . . . . . . . 21

Performances in High Magnetic Fields of Fine-mesh Photomultipliers for Fast Time-of-flight Detectors . . . . . . . . . . . . . 26

Recent Results on GridPix Detectors: An Integrated Micromegas Grid and a Micromegas Ageing Test . . . . . . . . . . . . . 31

RICH Detector at Jefferson Lab, Design, Performance and Physics Results . . . . . . . . . . . . . . . . . 36

Hybrid-Photon-Detectors in the LHCb RICH System . . . . . . . 42

Development of a Fast Transition Radiation and Tracking Detector for CBM at FAIR . . . . . . . . . . . . . . 47

R\&D on a Detector for Very High Momentum Charged Hadron Identification in ALICE . . . . . . . . . . . . . . 54

The Design and Test of the ATLAS Diamond Beam Conditions Monitor . . . . . . . . . . . . . . . . . 60

The AMS02 Transition Radiation Detector (TRD) - A Gasfilled Detector for the International Space Station . . . . . . . . . 66

A New Automatic Microscope for High Speed Analysis of Nuclear Emulsions

A Novel Type of Proximity Focusing RICH Counter with Multiple Refractive Index Aerogel Radiator . . . . . . . . . . . . 77

A Subminiature Scintillation Detector for Catheter Operation $\ldots 82$ Analysis of Test-beam Data from a Prototype LHCb RICH Detector 87 Single Crystal CVD Diamond Detectors for Hadron Physics . . . . . 92 Improvement of Particle Identification by Energy Loss in a Stack of Silicon Detectors . . . . . . . . . . . . . . . . . 98

Studies for a Fast RICH . . . . . . . . . . . . . . . . . 103

Properties of Scintillator Strip with Wavelength Shifting Fiber and Silicon Photomultiplier 
Astroparticle Experiments $\ldots \ldots \ldots \ldots$

The Underground Baradello Laboratory: Characterization of the Site and Early Results on Gamma-Ray and Neutron Spectrometry

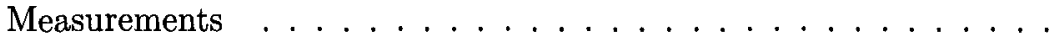

The Art of Detecting Neutrinos, $2 \mathrm{~km}$ Underground - The Three Phases of the Sudbury Neutrino Observatory . . . . . . . . . . . . 122 Neutrino Physics at the South Pole - Recent results from the AMANDA Experiment

First Results from GLAST-LAT Integrated Towers Cosmic Ray Data Taking and Montecarlo Comparison . . . . . . . . . . . . Background Analysis of Cuoricino in View of the Future Experiment CUORE

Application of Nuclear Track Detectors in Astroparticle and Nuclear Physics

Recent DAMA Results

Double Beta Decay Measurement with COBRA . . . . . . . . 168

Ultraviolet Diamond Photodetector . . . . . . . . . . . . . 173

Status of the KATRIN Experiment $\ldots \ldots \ldots \ldots \ldots$

The RPC System for the OPERA Spectrometers . . . . . . . . . 185

Electromagnetic Shower Reconstruction in Emulsion Cloud Chamber 190

A Low Background Micromegas Detector for the CAST Experiment 196 Imaging Optical Adapters for Multi-Anode Photo Multipliers Detectors . . . . . . . . . . . . . . . . . . . . . . 201

A 2D Stochatsic Montecarlo for the Solar Modulation of GCR: A Procedure to Fit Interplanetary Parameters Comparing to the Experimental Data . . . . . . . . . . . . . . . . . . 206 Spatial Distribution of Energetic Heavy Ions near the Earth . . . 212 A Photon Tag Calibration Beam for the AGILE Satellite . . . . . 217 Observation Program of Isotope Composition in the Ultra Heavy Cosmic Rays . . . . . . . . . . . . . . . . . . . . High-Energy Cosmic Rays Investigated by Air-shower Measurements with KASCADE, KASCADE-Grande, and LOPES

Scintillating Optical Fibers for Astroparticle Physics . . . . . . . . . The T2K Experiment: Status and Instrumentation of the $280 \mathrm{~m}$ Near Detector . . . . . . . . . . . . . . . . . . . 245

Results and Status of the PICASSO Experiment . . . . . . . 253

Data Acquisition System and Trigger Electronics for CACTUS . . . 262

The Central Pixel of the MAGIC Telescope for Optical Observations 267

Status report of ARDM Project: a New Direct Detection Experiment, Based on Liquid Argon, for the Search of Dark Matter 
Development of Multiple Photon Counting Coincidence (MPCC) Technique for Characterisation of Scintillators for Cryogenic Applications Results from Cuoricino Experiment and Prospects for CUORE . . . Timing Calibration of the NEMO Optical Sensors . . . . . . . . . 292 Design, Production, and First Results from the IceCube Digital Optical Module

\section{Calorimetry}

Study of Electromagnetic Calorimeter Trigger-Primitive Performances for CMS Data Selection . . . . . . . . . . . . . . . 307

The CMS Electromagnetic Calorimeter . . . . . . . . . . . 312 The CMS ECAL Laser Monitoring System . . . . . . . . . . 318 Pointing Calorimeter for Measuring $K_{L}^{0} \rightarrow \pi^{0} \nu \bar{\nu}$ Decay and Development of Extruded Scintillator . . . . . . . . . . . . . . . . . 323 CMS Preshower In-situ Absolute Calibration . . . . . . . . . . . 328 Dedicated Front-End Electronics for an ILC Prototype Hadronic Calorimeter with SIPM readout

Using Single Photoelectron Spectra in the Calibration of the CMS-HF Calorimeter . . . . . . . . . . . . . . . . . . . . 338 Jet Energy Scale Determination for the Run II D $\emptyset$ Calorimeter . . . 343 The Hadron Calorimeter of the CMS Experiment at the Large Hadron Collider

Properties of a 256-channel Prototype of the ALICE/PHOS Calorimeter . . . . . . . . . . . . . . . . . 353

Source Calibration of the CMS Quartz Fiber Forward Calorimeter . 358 The Calibration Strategy of the CMS Electromagnetic Calorimeter . 363 The Very Front-End Cards for the CMS Electromagnetic Calorimeter: Description, Calibration and Performance . . . . . . . . . . Uniformity of Response of the ATLAS Electromagnetic Calorimeter Series Modules

Plans for the Very Forward Region of ATLAS - the LUCID

Luminosity Monitor . . . . . . . . . . . . . . . . . . . . 379

Fast, Long-Wavelength Scintillators and Waveshifters . . . . . . 389

Test Beam Results of the CMS Electromagnetic Calorimeter . . . . 395

Electron and Pion Results from the ATLAS Foward Calorimeter 2003 Test Beam

Linearity, Energy and Position Resolution of the ATLAS Electromagnetic Calorimeter Series Modules . . . . . . . . . . . . . . . Preshower Silicon Strip Detectors for the CMS Experiment at LHC 
Inter-Calibration and Offline Compensation Studies of the ATLAS Calorimeter Using Beam Tests and Monte Carlo . . . . . . . . . . . 421

GEANT4 and Software Applications . . . . . . . . 425

Symposium on the Applications of the GEANT4 Simulation Software 427 GEANT4 Applications for NASA Space Missions . . . . . . . . . . 429 GEANT4 Application to Hadrontherapy in Japan . . . . . . . . . 437 AMS02 Italian Data Transfer System: Real Life Experience . . . . . 444 Application of GEANT4 in the Development of New Radiation Therapy Treatment Methods . . . . . . . . . . . . . . . . 451 G4 Accelerator Applications . . . . . . . . . . . . . . . 462 A Full Monte Carlo Simulation of Silicon Strip Detectors . . . . . 470 Simulation of Radiation Monitors for Future Space Missions . . . 475 Electromagnetic Physics Modeling in GEANT4 Package . . . . . . . 480 Perspectives in Medical Applications of Monte Carlo Simulation Software for Clinical Practice in Radiotherapy Treatments . . . . 485 GEANT4 Monte Carlo Simulation to estimate Gamma-Ray Emissions from Lunar Surface and SELENE Spacecraft . . . . . . . . . . . . CORAL, a Software System for Vendor-Neutral Access to Relational Databases

Track Extrapolation with Intrinsic Navigation in the New ATLAS Tracking Scheme . . . . . . . . . . . . . . . . 506 Applications of GEANT4 for the ESA Space Programme . . . . 511 Detector Simulation in High Energy Physics . . . . . . . . . . 519

High Energy Physics $\ldots \ldots \ldots \ldots \ldots$

Fast Shower Parametrisation for Electromagnetic Cascades . . . . . 533 Technical Aspects of the Manufacture of 4 Grease Pad Assemblies for the CMS Experiment

Implementation and Performance of a Tau Lepton Selection within the ATLAS Trigger System at the LHC

ATLAS Detector Simulation: Status and Outlook . . . . . . . . 551

The ATLAS Experiment at CERN: Two Years Before the Start of the Data Taking

Progress in Grid Computing for Particle Physics . . . . . . . . . . 565

Design, Production and Quality of the Sensors for the Silicon Strip Tracker of CMS 
ATLAS Inner Detector Results from the 2004 Combined Test Beam Data

The CMS Muon System . . . . . . . . . . . . . . . . . . 605

Performances of the ATLAS Level-1 Muon Trigger Processor in the Barrel Certification, Installation and Commissioning of Muon Drift Tube Chambers for the CMS Central Muon Detector . . . . . . . . . . The LHC Collider: Status and Outlook to Operation

PANDA: A Detector for Research with Antiprotons

Test of QED with the Reaction $e^{+} e^{-} \rightarrow \gamma \gamma(\gamma)$

Muon Reconstruction and Identification for the Event Filter of the ATLAS Experiment . . . . . . . . . . . . . . . . . . . . . . 648

\section{Medical Applications and Instrumentations}

Spectral and Spatial Distribution of the Scattered Radiation of an Electron Radiotherapy Beam . . . . . . . . . . . . . . . 655

A Liquid Ionization Chamber as Monitor in Radiotherapy . . . . . . 661 The Mammographic Head Demonstrator Developed in the Framework of the "IMI" Project: First Imaging Tests Results . . . . . . . . . . 666 Optimized Infrared Detectors for Infrared Synchrotron Radiation Microspectroscopy and Biomedical Imaging . . . . . . . . . . . MATRIX: An Innovative Pixel Ionization Chamber for On-line Beam Monitoring in Hadrontherapy . . . . . . . . . . . . . . Development of a Dual Tracer PET Method for Imaging Dopaminergic Neuromodulation

Rethinking Positron Emission Technology for Early Cancer Detection 692 Positron Emission Tomography - Tracer Kinetic Modelling in Drug Development . . . . . . . . . . . . . . . . . 3D-Reconstruction of Absorbed Dose Obtained from Gel-DosimeterLayers . . . . . . . . . . . . . . . . . . . . . . . 705 Accurate Determination of Radionuclidic Purity and Half-Life of Reactor Produced Lu-177g for Metabolic Radioimmunotherapy . . . 710 Spatial Linearity Improvement for Discrete Scintillation Imagers . . 715 High Resolution, High Sensitivity Detectors for Molecular Imaging of Small Animals and Tumor Detection

Strip Ionization Chamber as Beam Monitor in the Proton Therapy Eye Treatment

Low Dose, Low Energy 3D Image Guidance During Radiotherapy . 732 Alpha Cyclotron Production Studies of the Alpha Emitter ${ }^{211} \mathrm{At} /{ }^{211}{ }^{11} \mathrm{Po}$ for High-LET Metabolic Radiotherapy 
Treatment Planning with IVIS Imaging and Monte Carlo Simulation

Monte Carlo Simulations of a Human Phantom Radio-Pharmacokinetic Response on a Small Field of View Scintigraphic Device . . . . . . .

Applications of the Monte Carlo Code GEANT to Particle Beam Therapy

Charge Sharing in Pixel Detectors for Spectroscopic Imaging

Direct Thickness Calibration: Way to Radiographic Study of Soft Tissues

A portable Pixel Detector Operating as an Active Nuclear Emulsion and Its Application for X-Ray and Neutron Tomography ......

\section{Radiation Damage}

Statistical Study of Radiation Hardness of CMS Silicon Sensors . . SIC $\mathrm{PbWO}_{4}$ Crystals for the Electromagnetic Calorimeter of CMS Experiment

MDT Chamber Ageing Test at ENEA Casaccia Neutron and Gamma Facilities

Behavior of Thin Film Materials Under $\gamma$ Irradiation for Astronomical Optics

Full Characterization of Non-Uniformly Irradiated Silicon Micro-Strip Sensors

Beam Energy Monitor for 4-10 MeV Electron Accelerators

An Analysis of the Expected Degradation of Silicon Detectors in the Future Ultra High Energy Facilities

Investigation of VLSI Bipolar Transistors Irradiated with Electrons, Ions and Neutrons for Space Application

Radiation-Hardness Studies of High $\mathrm{OH}^{-}$Content Quartz Fibres

Irradiated with $24 \mathrm{GeV}$ Protons

Development of Radiation Hard Silicon Detectors: The SMART Project

Scintillator and Phosphor Materials: Latest Developments and Applications

Crystals for High-Energy Physics Calorimeters in Extreme

Environments 
Photoluminescence and $\gamma$-Ray Irradiation of SrO- $\mathrm{B}_{2} \mathrm{O}_{3}-\mathrm{P}_{2} \mathrm{O}_{5}: \mathrm{Eu}^{2+}$ and $\mathrm{SrMoO}_{4}: \mathrm{Eu}^{3+}$ Phosphors . . . . . . . . . . 883

\section{Space Experiments}

The Extreme Universe . . . . . . . . . . . . . . . . . . 891

PAMELA Data Acuisition System . . . . . . . . . . . 898

AGILE MCAL, the Mini-Calorimeter . . . . . . . . . . . . 904

Primary Helium CR Inside the Magnetosphere: A Transmission

Function Study . . . . . . . . . . . . . . . . . . . 909

Lanthanum Halide Scintillators and Optical Fiber Readout for XRay/Gamma-Ray Astronomy and National Security Applications . 917

The AGILE Silicon Tracker: Construction and Calibration Results . 922 Ions Abundance Close to the Earth Surface: The Role of the Magnetosphere . . . . . . . . . . . . . . . . . . . . 928

Design of a Silicon Transition Radiation Detector (SiTRD) for Accelerators and Space Applications . . . . . . . . . . . . 935

The Origin of Helium-3 Isotope Enhancement in the Magnetosphere Observed by TSUBASA Satellite . . . . . . . . . . . . . . 940

The Microscope Mission and Pre-Flight Performance Verification . . 945 Performance of the Integrated Tracker Towers of the GLAST Large Area Telescope . . . . . . . . . . . . . . . . . . . . 952

Performance of Neutron Detector and Bottom Trigger Scintillator of the Space Instrument PAMELA . . . . . . . . . . . . High Granularity Silicon Beam Monitors for Wide Range Multiplicity

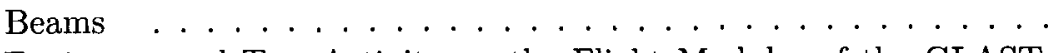
Environmental Test Activity on the Flight Modules of the GLAST LAT Tracker . . . . . . . . . . . . . . . . The Time of Flight Detector and Trigger for the PAMELA Experiment in Space . . . . . . . . . . . . . . . . 973

Fundamental Physics in ESA's Cosmic Vision Plan . . . . . . 979

The AMS-02 Electronics System . . . . . . . . . . . . . . . . 989

Launch in Orbit of the Space Telescope PAMELA and Ground Data Results . . . . . . . . . . . . . . . . . . . . . . . 994

CZT Detector Development for New Generation Hard-X/GammaRay Astronomical Instruments . . . . . . . . . . . . . .

Tracking Devices

A Barrel IFR Instrumented with Limited Streamer Tubes for BaBar Experiment . . . . . . . . . . . . . . . . 
A New Inner Layer Silicon Strip Detector for D0 . . . . . . . . 1012 Calibration System for the Silicon Drift Detector of the ALICE Experiment . . . . . . . . . . . . . . . . . Experience with the Resistive Plate Chamber in the BaBar Experiment

Muon Identification and Reconstruction in the ATLAS Detector at the LHC

Micrometric Position Monitoring Using Fiber Bragg Grating Sensors in Silicon Detectors

Commissioning of the CMS Tracker Outer Barrel . . . . . . . . . .

New Effects Observed in the BaBar Silicon Vertex Tracker: Interpretation and Estimate of Their Impact on the Future Performance of the Detector

Tests of Substructures of the CMS Silicon Strip Tracker . . . . . .

Test, Qualification and Electronics Integration of the ALICE Silicon Pixel Detector Modules . . . . . . . . . . . . . . . . . . 1054

Layout and Status of the CMS Silicon Tracker . . . . . . . . 1060

The CMS-Tracker Detector Controls System . . . . . . . . . 1067

Optimization of the Readout Pad Geometry for a GEM-based Time Projection Chamber . . . . . . . . . . . . . . . . . . . . Tracking Strategy and Performance for the ATLAS High Level Triggers . . . . . . . . . . . . . . . . . . . . 1077

The LHCb Silicon Tracker _. . . . . . . . . . . . . . . 1082

The H1 Silicon Tracker . . . . . . . . . . . . . . . . . . . . 1087

The LHCb Muon System . . . . . . . . . . . . . . . . . . . . 1092

Two- and Three-Dimensional Reconstruction and Analysis of the Straw Tubes Tomography in the BTeV Experiment . . . . . . . . . . . . 1098

Cryogenic Operation of Edge-Sensitive Silicon Microstrip Detectors 1104 The DEPFET Active Pixel Sensor as Vertex Detector for the ILC . 1109 Final Assembly and Intergration of the ATLAS Semiconductor Tracker and Transition Radiation Tracker

3-Dimensional Position Control for the AMS-02 Tracker with Infrared Laser Beams

Development of a GEM-based High Resolution TPC for the

International Linear Collider

STAR Inner and Forward Tracking Upgrade

List of participants 